\title{
Wavelength assignment in optical networks according to traffic requirements and transmission impairments
}

\author{
M. Moreschini, F. Matera, M. Settembre \\ Fondazione Ugo Bordoni \\ via B. Castiglione 59, 00142 Roma, Italy \\ tel.+39-06-54802215, fax+39-06-54804402,e-mail mat@fub.it
}

Key words: networks, optical systems, FWM, XPM, routing, WDM, wavelength

\begin{abstract}
We describe a novel algorithm that designs wavelength-routed optical telecommunication networks taking into account both the traffic demands and the physical limits due to the WDM signal transmission. Some results for a metropolitan ring are reported.
\end{abstract}

\section{INTRODUCTION}

The explosion of the Internet phenomenon shows that in the next years telecommunication networks will operate with Terabits/s capacities. Optical technology can satisfy such a requirement thanks to its advances and in particular to the development of the Wavelength Division Multiplexing (WDM) technique. At the moment it is accepted that the novel high capacity networks should be based on wavelength routing devices [1]. This way all the traffic from node $i$ to node $j$ can be carried by directly using one or more dedicated wavelengths. However due to some physical limitations and cost reasons, the number of the wavelengths could be insufficient to obtain all the direct optical paths that are required by the traffic requirement, so electronic mu-demultiplexing is needed to allow low rate nodes to share the same wavelength. As a consequence, for a network design, traffic grooming and wavelength assignment algorithms are required. At the moment several algorithms have been already proposed, but at our knowledge, none of these 
takes into account the physical impairments of the networks, especially the ones due to the signal propagation. Also for metropolitan networks the fibre impairments could result much degrading for the WDM transmission and in particular the Kerr nonlinearity can manifest as a strong limitation both in the G.652 fibres in terms of Cross Phase Modulation (XPM) and in G.653 in terms of Four Wave Mixing (FWM) [3-4].

The aim of this work is to show an algorithm that permits to assign the wavelengths in an optical networks taking into account both the traffic requirements and the propagation limitations. Such an algorithm is used to design a metropolitan optical network connecting seven nodes by means of a fibre ring considering different values of the total traffic.

\section{ALGORITHM}

Our algorithm, based on the one reported in ref. [1], permits to achieve a matrix [W] describing the wavelength connections among the nodes taking into account traffic demand and physical impairments. We suppose that each node has some electronic Add Drop Multiplexers (ADMs) [2] and a Wavelength ADM (WADM). Each ADM is used to aggregate low rate signals onto a single high rate signal that is carried on a wavelength representing a path. In each node the wavelengths can be mu-demultiplexed by means of the WADM, and if the information carried by a dropped wavelength is not directed to the node it is retransmitted on other wavelength.

First of all we define the following input network parameters: the traffic matrix [T], the channel bit rate $\mathrm{R}$, the number of nodes, $\mathrm{N}$, and the physical parameters of the links connecting the nodes.

The algorithm mainly consists of two parts: the transmission and traffic routine.

Transmission routine. Analytical calculations permits in many propagation cases to evaluate the maximum capacity versus distance. In the presence of FWM and XPM the maximum number of wavelength, $\mathrm{w}_{\mathrm{MAX}}$, versus distance, can be analytically evaluated by using the methods reported in ref. [3] that permit to evaluate the $Q$ factor. We remember that to have a bit error rate lower than 10-9, under some hypothesis [4], the Q factor has to be higher than 6 . We calculate the number $\mathrm{w}_{\operatorname{MAX}}$ versus distance by assuming the condition that each channel has to assume a $Q>6$ in a power interval between Pmin and 10 Pmin, where Pmin is the minimum power that permits to overcome to the noise effects. 
The function $\mathrm{w}_{\operatorname{MAX}}\left(\mathrm{n}_{\mathrm{c}}\right)$, where $\mathrm{nc}$ is the number of crossed node, is used as constraint of the traffic routine.

Traffic routine. This routine is composed by some steps that consists in finding the logical topology that permits to route the traffic with $1,2,3$. hops and each time we verify if the wavelength distribution in each fibre link can be permitted by the link limitations that have been previously evaluated.

First of all we calculate the matrix [W(0)] whose elements $\mathrm{w}(0) \mathrm{ij}$ are given by the integer number corresponding to [tij/R]. Starting from [W(0)], we can calculate for each wavelength, $s$, the number of wavelengths, nmet$\mathrm{s}\left(\mathrm{n}_{\mathrm{c}}\right)$, that are met during its path. By analysing previous simulations we have observed that the degradation of each wavelength path can be taken into account by defining the function, $\mathrm{nW}-\mathrm{s}\left(\mathrm{n}_{\mathrm{c}}\right)$, obtained by arranging in a decreasing order the values nmet-s; a correct design of the network is obtained when for each $s$ and $n c$ the value of $n W-s$ is always lower than $\mathrm{W}_{\text {MAX. }}$.

If such a condition is not verified the wavelength assignment will be changed looking for a new configuration taking into account the requirement of $\mathrm{w}_{\operatorname{MAX}}\left(\mathrm{n}_{\mathrm{c}}\right)$. By means of this procedure, if the physical conditions are compatible with the traffic requirement, a matrix [W(0)] will be obtained.

After this step we can find the wavelength assignment for the remaining traffic $[\mathrm{T}(1)]=[\mathrm{T}]-\mathrm{R}[\mathrm{W}(0)]$. In particular according to ref. [1] the assignment can be obtained by solving the following simple linear program

$$
F(h 1)=\max \sum_{i=1}^{N} \sum_{j=1}^{N} w_{i j}{ }^{(1)} T^{(1)} i j \quad i \neq j
$$

where $\operatorname{wij}(1)=1$ if a wavelength is available between $i$ and $j$ ( 0 otherwise) and by considering some constraints.

We have assumed two constraints, the first is that each node always has at least two input-output wavelengths and that the total number of wavelength has to be lower than a value $\mathrm{M}_{\mathrm{hl}}$. As in the previous step, the wavelength assignment has to satisfy the physical conditions.

At this point if all the traffic has been not routed we will continue with further steps considering other hops and assuming other values $\mathrm{M}_{\mathrm{h} 2}, \mathrm{M}_{\mathrm{h} 3 \text {.., }}$, up to finish all the traffic in agreement with the physical requirements. In particular, in each step, first we try to encapsulate the traffic in the wavelengths that have available circuits (155 Mb/s, STM-1) and subsequently for the remaining traffic we consider another simple linear program as reported in ref. [1]. For a fixed number of hops, to have an efficient wavelength assignment, the values $\mathrm{Mh}$ have to be minimised. 
At the end of this routine, if the transmission conditions are compatible with the traffic demand, we will have the final matrix $[\mathrm{W}]=[\mathrm{W}(0)]+$ $[\mathrm{W}(1)]+[\mathrm{W}(2)]+.$.

\section{RESULTS}

We have used such an algorithm to design an optical WDM ring network that connects seven high capacity nodes. The optical part of each node consists of a WADM and an EDFA amplifier that compensates for the fibre loss $(0.25 \mathrm{~dB} / \mathrm{km})$ and for the other loss of the node devices $(10 \mathrm{~dB})$. The total length of the ring is $42 \mathrm{~km}$, the average distance among the nodes is 7 $\mathrm{km}$. Such a physical structure is compatible with the geographical distribution of the main telecommunication centres of Rome. We assume that the ring, composed by two fibres (one is for providing protection), can encompass both G.652 and G.653 fibres. For G.562 fibres the chromatic dispersion is $16 \mathrm{ps} / \mathrm{nm} / \mathrm{km}$, while for G.653 fibre the chromatic dispersion is $1 \mathrm{ps} / \mathrm{nm} / \mathrm{km}$ in the centre of the WDM signal bandwidth. We have assumed a traffic matrix $[\mathrm{T}]=\Psi[\Gamma]$, where $\Psi$ is a coefficient ranging between 1 and 10 , and $[\Gamma]$ is a $7 \times 7$ matrix whose row elements are: $0,20,5,8,8,8,6 ; 20,0,15$, $30,22,10,3 ; 5,10,0,6,6,6,7 ; 8,11,5,0,8,8,7 ; 9,10,5,8,0,8,10 ; 10$, $10,4,8,8,0,9 ; 7,2,6,9,9,9,0$. According to these data, the total traffic of the network varies between 38.3 and $383 \mathrm{~Gb} / \mathrm{s}$. We assume a channel bit rate equal to $10 \mathrm{~Gb} / \mathrm{s}$ with a frequency spacing among the channel of $50 \mathrm{GHz}$ and optical amplifiers with a flat gain in a bandwidth of $30 \mathrm{~nm}$.

In these propagation condition, in G.652 fibres, XPM and FWM have a negligible contribution and also the pulse broadening due to the chromatic dispersion is not relevant. In G.653 links FWM for can induce a relevant degradation and this is shown by the function $\mathrm{w}_{\mathrm{MAX}}$ that assumes the values of $72,27,12$ and 4 for nc equal to 3, 4, 5 and 6 respectively. It means that for G.652 links the wavelength assignment is only given by the traffic algorithm, while for G.653 links the transmission routine has a relevant role. In particular for G.653 links, the function $\mathrm{w}_{\mathrm{MAX}}$ shows as the propagation of many wavelengths having long paths should be avoided and it suggests to decrease the length of the paths at expense of an increasing of the wavelengths. In our cases all the traffic can be routed by considering at maximum 5 hops (h5).

In fig. 1 we report the required number of wavelengths versus the total traffic considering 1 (h1), 2 (h2) and 5 hops. In the G.652 fibres, in the h1 case, the number of wavelengths is high for any traffic condition, since each node has to be connected to all the other nodes. Conversely, when the traffic is high the difference between $\mathrm{h} 1$, and $\mathrm{h} 5$ is not so relevant since many 
wavelengths are required to carry the heavy traffic. For G.653 links due to the values of $\mathrm{w}_{\mathrm{MAX}}$, the configuration with $\mathrm{h} 1$ cannot be used, and, especially for high traffic, a larger number of wavelengths is required with respect to the G.652 case, to avoid the presence of many long wavelength paths. However too wavelengths can induces a high FWM effect limiting the wavelength assignment as shown in fig. 1 for $\mathrm{h} 2$ for a traffic higher than 250 $\mathrm{GHz}$.

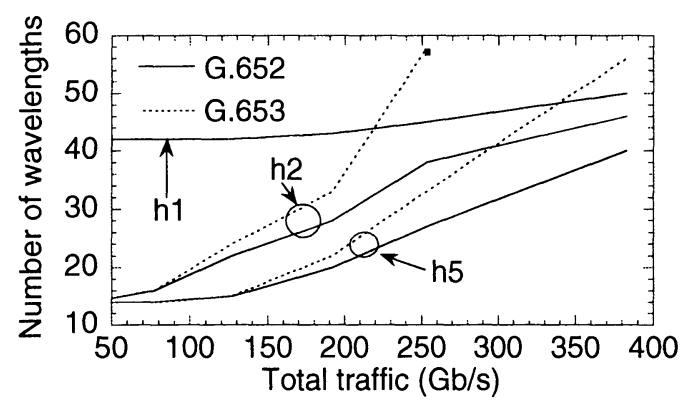

Fig. 1: Number of wavelengths vs total traffic for the optical ring network with $\mathrm{R}=10 \mathrm{~Gb} / \mathrm{s}$.

The problems of the network encompassing G.653 fibres can be overcome by doubling the fibres and introducing two counterpropagating directions in the rings; this way the wavelength is transmitted on the ring with the direction that permits to have the shortest path between the nodes.

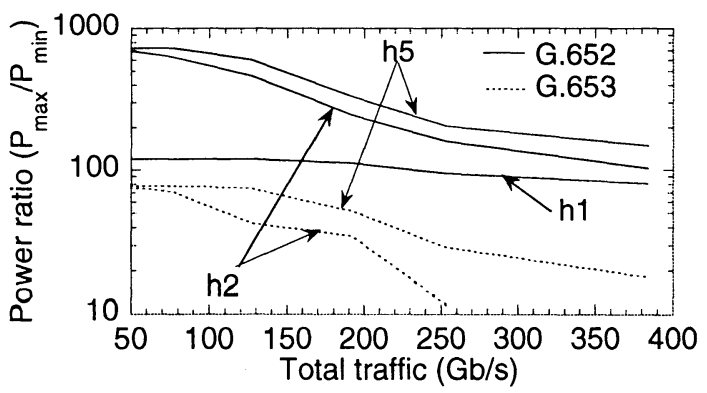

Fig. 2: transmission tolerance in terms input power per channel with the wavelength assignment of fig. 1 . 
The transmission performance of the network have been tested by means of a simulation code based on the split step method. For each network configuration we have evaluated the $Q$ factor versus input power for each wavelength path; in conditions of good performance $Q$ is higher than 6 a power interval between Pmin and Pmax, where Pmax is the maximum input power permitted by the nonlinear effects. Among all the paths the worst ratio $\rho=P \max / \mathrm{Pmin}$ is found and it is reported in fig. 2 . The figure 2 shows how the performance decreases by increasing the traffic and that, for the considered network, a better transmission quality can be achieved by using the G.652 fibres.

The results shown in fig. 1 for G.652 fibres can be used for a network with the same physical parameters, a lower channel bit rate and a reduced traffic; for an instance the same wavelength assignment can be obtained for a channel bit rate of $2.5 \mathrm{~Gb} / \mathrm{s}$ and a total traffic ranging between 9.6 and 96 $\mathrm{Gb} / \mathrm{s}$.

\section{CONCLUSIONS}

We have described an algorithm that permits to assign the wavelengths in an optical network taking into account both the traffic and transmission requirements. As an example we have reported the results obtained for a metropolitan network in which the FWM effect can show severe impairments.

\section{ACKNOWLEDGEMENTS}

Work carried out under the agreement between Fondazione Ugo Bordoni and Italian Communications Administration (ISCTI) and in the framework of the European COST266 and of the IST ATLAS project.

\section{REFERENCES}

[1] S. Banerjee, J. Yoo, C. Chen, "Design of wavelength-routed optical networks for packet switched traffic" J. of Lightwave Technology, 15, 1636-1646 (1997).

[2] A. L. Chiu, E. H. Modiano, "Traffic grooming algorithms for reducing electronic multiplexing costs in WDM rings networks" J. of Lightwave Technology, 18 , 2-12 (2000)

[3] S. Ten, K. M. Ennser, J. M. Grochocinski, S. P. Burtsev, V. L. daSilva, "Comparison of four-wave mixing and cross phase modulation penalties in dense WDM systems" Proc. of OFC'99, San Diego (CA), ThC4-1/43 (1999).

[4] F. Matera, M. Settembre, Journal of Lightwave Technology, "Comparison of the Performance of Optically Amplified Transmission Systems” 14, 1-12 (1996). 